\title{
The Role of Functional Head Impulse Test With and Without Optokinetic Stimuli in Vestibular Migraine and Acute Unilateral Vestibulopathy: Discovering a Visual Dependence?
}

\author{
Augusto-Pietro Casani ( $\nabla$ augusto.casani@unipi.it ) \\ Pisa University Hospital, Università di Pisa, Italy \\ Francesco Lazzerini \\ Pisa University Hospital, Università di Pisa, Italy \\ Nicola Ducci \\ Pisa University Hospital, Università di Pisa, Italy \\ Ottavia Marconi \\ Pisa University Hospital, Università di Pisa, Italy \\ Nicola Vernassa \\ Pisa University Hospital, Università di Pisa, Italy
}

\section{Research Article}

Keywords: Functional Head Impulse, AUV, Vestibular Migraine

Posted Date: April 27th, 2021

DOI: https://doi.org/10.21203/rs.3.rs-449538/v1

License: (c) (i) This work is licensed under a Creative Commons Attribution 4.0 International License.

Read Full License 


\section{Abstract}

Visually induced vertigo (i.e., vertigo provoked by moving visual scenes) could be considered as a noticeable feature of Vestibular Migraine (VM) and could be present in patients suffering from Acute Unilateral Vestibulophaty (AUV). The hypersensitivity to moving or conflicting visual stimulations is named Visual Dependence. The visuo-vestibular interactions were analyzed using functional Head Impulse Test (fHIT) with and without optokinetic stimulation (o-fHIT) in 25 patients with VM and 20 subjects affected by AUV. We calculated the percentage of correct answer (\%CA) without and with the addition of confounding screen (CS). In VM groups the \%CA at fHIT was $92,07 \%$ without CS and $73,66 \%$ with CS. A significant difference has been found between \%CA on the deficit side and the normal side in AUV, both without CS and with CS). The results of fHIT in term of \%CA with and without CS could be very useful to identify the presence of visual dependence especially in patients suffering from VM. The difference of \%CA with and without CS could provide an instrumental support to correctly identify patients with VM. We propose the use of fHIT in clinical practice whenever there is a need to highlight a condition of visual dependence.

\section{Introduction}

Vertigo and dizziness are common complaint requiring an accurate clinical and instrumental assessment. In otoneurological practice, Acute Unilateral Vestibulopathy (AUV) and Vestibular Migraine (VM) represent two broad categories of vestibular disorders: AUV is the most important cause of sustained vertigo characterized by a sudden onset of long-lasting vertigo with nausea and vomiting, gait instability, and a falling tendency toward the affected side without associated cochlear or central nervous system symptoms and signs [1]. VM is now considered as the most common cause of episodic vestibular syndrome: a 1-year prevalence has been estimated at $2.7 \%$ in a population-based study [2] a much higher number as previously reported [3]. Patients suffering from these two conditions could present in different stages of the disease some degree of visuo-vestibular symptoms: AUV patients could develop chronic symptoms such as head movement and visually induced dizziness, especially during head turns [4]. Patients with VM could manifest, along with various types of vertigo (spontaneous vertigo, positional vertigo), visually induced vertigo, head motion-induced vertigo, and head motion- induced dizziness with nausea $[5,6]$. Visually induced vertigo (i.e., vertigo provoked by moving visual scenes, such as traffic or movies) could be considered as a noticeable feature of VM and may persist between attacks [7]. The hypersensitivity to moving or conflicting visual stimulations is named "Visual Vertigo" or "Visual Dependence" (VD): the subject with VD privileges the degree of visual reliance during central integration of sensory cues and this condition reduces the ability to disregard visual clues in complex or conflicting visual environments [8]. The exact reason of the over-reliance in visual stimuli is still matter of debate $[9,10]$; however, both AUV (probably because of an increase in visual contribution in the sensory integration process) and VM (a reduced capability to integrate different sensory signals reporting motion has been observed) [11-13] could facilitate the onset of symptoms compatible with VD [10]. 
In order to assess and quantify the role of VD in different physiological and pathological conditions, the Rod and Frame and the Rod and Disc techniques $[4,11,14]$ as well as the measurement of the postural sway (induced by visual-roll-motion stimulation) by static posturography [4] have been used. Recently, a new test offered the possibility to obtain precise information about the patient's ability to achieve a clear vision during rapid head turns. This test was named functional Head Impulse Test (fHIT) and allows to obtain objective data not only about the gaze stabilization but also it is capable to detect the role of visual component in maintaining balance using a moving background while the test is administered $[15,16]$. The comparison of the responses without and with confounding background (optokinetic stimulation, o-fHIT) could give an indication of the effect on the ability to respond, expected much poorer in case of discrepancy between perceived visual stimulus and vestibule-proprioceptive stimulus $[13,15]$.

The aim of this paper was to evaluate the potential visuo-vestibular interactions in two groups of patients suffering respectively from AUV and VM, analyzing the results of fHIT both without and with optokinetic stimulation.

\section{Results}

Demographic data, clinical feature, and distribution of \%CA for the three evaluated groups is reported in Table 1 (patients with VM), table II (patients with AUV) and table III (control group, normal subjects). The mean age of the AUV group (59,6 years, ranging from 39 to 78, 11 males and 9 females) was slightly higher than VM (53,4 years, ranging from 32 to 67,15 females and 5 males). The control, group was constituted by 20 healthy subjects ( 10 males and 10 females, age ranging from 39 to 70 , mean 53,8). Regarding the patients suffering from VM, the duration of symptoms was $9.8 \pm 6.5$ months (range 6-31). All the patients reported episodic attacks of rotational vertigo; 9 subjects suffered from chronic unsteadiness. 16 patients reported head motion intolerance and 20 suffered from motion sickness. Regarding the instrumental examination, we found a caloric hyperreflexia in 8 patients. Five patients showed a positional persistent nystagmus, in 1 case associated to the positivity of Head Shaking Test. 
Table 1

Vestibular migraine group (VM): demographic data, clinical features, distribution of percentage of correct answers (\%CA) on the left and on the right side, without and with confounding screen (CS), situational vertigo questionnaire (SVQ) score. $\mathrm{EV}=$ episodic vertigo; $\mathrm{DIZZ}=$ dizziness; $\mathrm{MS}$ = motion sickness; $\mathrm{HMI}=$ head motion intollerance; $\mathrm{Hy}=$ hyperreflexia; Pos $\mathrm{Ny}=$ positional persistent nystagmus .

\begin{tabular}{|c|c|c|c|c|c|c|c|}
\hline $\begin{array}{l}\text { Sex, } \\
\text { Age }\end{array}$ & $\begin{array}{l}\% \text { CA on } \\
\text { the left }\end{array}$ & $\begin{array}{l}\% \text { CA on } \\
\text { the right }\end{array}$ & $\begin{array}{l}\% \text { CA on the } \\
\text { left + CS }\end{array}$ & $\begin{array}{l}\% \text { CA on the } \\
\text { right }+ \text { CS }\end{array}$ & SVQ & $\begin{array}{l}\text { Instrumental } \\
\text { Signs }\end{array}$ & Symptoms \\
\hline$F, 41$ & 100 & 85 & 80 & 100 & 28 & PosNy; Hy & $\begin{array}{l}\text { EV, Dizz, } \\
\text { MS, HMI }\end{array}$ \\
\hline$F, 59$ & 80 & 100 & 90 & 90 & 21 & none & $\mathrm{EV}, \mathrm{MS}$ \\
\hline$M, 43$ & 95 & 90 & 70 & 94,4 & 32 & $\mathrm{Hy}$ & $\mathrm{EV}, \mathrm{MS}$ \\
\hline$F, 61$ & 100 & 100 & 90 & 100 & 25 & none & EV \\
\hline$F, 44$ & 100 & 90 & 100 & 55,6 & 45 & Hy & $\begin{array}{l}\text { EV, MS, } \\
\text { HMI }\end{array}$ \\
\hline$F, 63$ & 100 & 100 & 65 & 76 & 34 & PosNy & $\begin{array}{l}\text { EV, Dizz, } \\
\text { MS, HMI }\end{array}$ \\
\hline$F, 41$ & 90 & 100 & 70 & 100 & 28 & none & $\begin{array}{l}\text { EV, Dizz, } \\
\text { MS, HMI }\end{array}$ \\
\hline$F, 46$ & 100 & 100 & 45 & 77,8 & 45 & Hy & $\begin{array}{l}\text { EV,MS, } \\
\mathrm{HMI}\end{array}$ \\
\hline$F, 69$ & 85 & 80 & 40 & 20 & 56 & Hy & $\begin{array}{l}\text { EV, MS, } \\
\mathrm{HMI}\end{array}$ \\
\hline$F, 42$ & 90 & 100 & 90 & 33,8 & 61 & none & $\begin{array}{l}\text { EV, Dizz, } \\
\text { MS, HMI }\end{array}$ \\
\hline M, 32 & 80 & 66,7 & 90 & 40 & 48 & none & $\begin{array}{l}\text { EV, MS, } \\
\mathrm{HMI}\end{array}$ \\
\hline$F, 44$ & 100 & 90 & 75 & 90 & 32 & PosNy & EV, Dizz \\
\hline$F, 55$ & 90 & 100 & 100 & 81,8 & 29 & none & $\begin{array}{l}\text { EV, Dizz, } \\
\text { MS, HMI }\end{array}$ \\
\hline$F, 51$ & 75 & 100 & 100 & 72,7 & 45 & none & $\begin{array}{l}\text { EV, MS, } \\
\mathrm{HMI}\end{array}$ \\
\hline M, 45 & 76,9 & 70 & 72 & 55,6 & 41 & $\begin{array}{l}\text { PosNy; } \\
\text { HST+ }\end{array}$ & EV, Dizz \\
\hline$M, 38$ & 100 & 100 & 65 & 100 & 25 & none & $\begin{array}{l}\text { EV, MS, } \\
\mathrm{HMI}\end{array}$ \\
\hline$F, 62$ & 80 & 100 & 65 & 82,4 & 39 & Hy & $\begin{array}{l}\text { EV, Dizz, } \\
\text { MS, HMI }\end{array}$ \\
\hline$F, 47$ & 100 & 90 & 80 & 22,2 & 51 & none & $\begin{array}{l}\text { EV, MS, } \\
\text { HMI }\end{array}$ \\
\hline
\end{tabular}




\begin{tabular}{|llllllll|}
\hline $\begin{array}{l}\text { Sex, } \\
\text { Age }\end{array}$ & $\begin{array}{l}\text { \% CA on } \\
\text { the left }\end{array}$ & $\begin{array}{l}\text { \% CA on } \\
\text { the right }\end{array}$ & $\begin{array}{l}\text { \% CA on the } \\
\text { left + CS }\end{array}$ & $\begin{array}{l}\text { \% CA on the } \\
\text { right + CS }\end{array}$ & $\begin{array}{c}\text { SVQ } \\
\text { Instrumental }\end{array}$ & $\begin{array}{l}\text { Symptoms } \\
\text { Signs }\end{array}$ \\
\hline F, 39 & 100 & 100 & 60 & 85 & 34 & none & EV, Dizz \\
\hline F, 38 & 90 & 100 & 80 & 66,7 & 52 & Hy & $\begin{array}{l}\text { EV, MS, } \\
\text { HMl }\end{array}$ \\
\hline F, 43 & 95 & 90 & 90 & 70 & 48 & none & EV, MS \\
\hline F, 57 & 70 & 100 & 50 & 80 & 46 & Hy & EV, MS, \\
\hline M, 43 & 90 & 100 & 85 & 90 & 28 & none & EV, MS \\
\hline F, 68 & 90 & 100 & 25 & 70 & 60 & Pos Ny & EV \\
\hline F, 41 & 85 & 90 & 65 & 86,7 & 56 & none & EV, MS, \\
\hline Mean & 90,47 & 93,66 & 73,68 & 73,62 & & & HMI \\
\hline
\end{tabular}


Table 2

Acute unilateral vestibulopathy group (AUV): demographic data, distribution of percentage of correct answers (\%CA) on the left (L) and on the right side (R), without and with confounding screen (CS), situational vertigo questionnaire (SVQ) score, asymmetry index (Al) detectable at vHIT.

\begin{tabular}{|c|c|c|c|c|c|c|}
\hline $\begin{array}{l}\text { Sex, } \\
\text { age }\end{array}$ & $\begin{array}{l}\% \text { correct } \\
\text { answers on } \\
\text { the left }\end{array}$ & $\begin{array}{l}\% \text { correct } \\
\text { answers on the } \\
\text { right }\end{array}$ & $\begin{array}{l}\% \text { correct } \\
\text { answers on the } \\
\text { left + CS }\end{array}$ & $\begin{array}{l}\% \text { correct answers } \\
\text { on the right + CS }\end{array}$ & SVQ & $\begin{array}{l}\text { VHIT } \\
\text { (AI) }\end{array}$ \\
\hline$M, 52$ & 60 & 100 & 40 & 80 & 28 & $\begin{array}{l}66 \% \\
\mathrm{~L}\end{array}$ \\
\hline$M, 53$ & 47,1 & 71,4 & 44,3 & 65,2 & 42 & $\begin{array}{l}70 \% \\
\mathrm{~L}\end{array}$ \\
\hline$F, 42$ & 50 & 90 & 45 & 83,4 & 39 & $28 \%$ \\
\hline$M, 52$ & 63,6 & 83,3 & 61,2 & 82,3 & 41 & $\begin{array}{l}55 \% \\
L\end{array}$ \\
\hline$F, 53$ & 70 & 90 & 20 & 41,7 & 43 & $\begin{array}{l}30 \% \\
\mathrm{~L}\end{array}$ \\
\hline$F, 57$ & 50 & 80 & 30 & 60 & 52 & $\begin{array}{l}41 \% \\
L\end{array}$ \\
\hline$M, 42$ & 60 & 90 & 40 & 70 & 28 & $\begin{array}{l}66 \% \\
L\end{array}$ \\
\hline$F, 62$ & 66,7 & 80 & 61,3 & 75,6 & 32 & $\begin{array}{l}56 \% \\
L\end{array}$ \\
\hline$M, 47$ & 30 & 90 & 20 & 75 & 37 & $28 \%$ \\
\hline$F, 54$ & 70 & 100 & 61,5 & 95 & 29 & $\begin{array}{l}38 \% \\
\mathrm{~L}\end{array}$ \\
\hline$F, 38$ & 22,2 & 63,6 & 19,5 & 55,9 & 53 & $22 \%$ \\
\hline Mean & 53,6 & 85,3 & 40,3 & 71,3 & & \\
\hline $\mathrm{F}, 70$ & 87,5 & 71,4 & 80,3 & 62 & 35 & $\begin{array}{l}41 \% \\
\mathrm{R}\end{array}$ \\
\hline $\mathrm{M}, 51$ & 88,9 & 40 & 88,9 & 39 & 32 & $\begin{array}{l}31 \% \\
\mathrm{R}\end{array}$ \\
\hline$M, 69$ & 90 & 70 & 80 & 50 & 31 & $\begin{array}{l}42 \% \\
\mathrm{R}\end{array}$ \\
\hline$M, 59$ & 40 & 12,5 & 35 & 10 & 51 & $\begin{array}{l}47 \% \\
\mathrm{R}\end{array}$ \\
\hline$F, 46$ & 90 & 40 & 88 & 32,6 & 28 & $\begin{array}{l}71 \% \\
\mathrm{R}\end{array}$ \\
\hline
\end{tabular}




\begin{tabular}{|c|c|c|c|c|c|c|}
\hline $\begin{array}{l}\text { Sex, } \\
\text { age }\end{array}$ & $\begin{array}{l}\% \text { correct } \\
\text { answers on } \\
\text { the left }\end{array}$ & $\begin{array}{l}\% \text { correct } \\
\text { answers on the } \\
\text { right }\end{array}$ & $\begin{array}{l}\% \text { correct } \\
\text { answers on the } \\
\text { left }+ \text { CS }\end{array}$ & $\begin{array}{l}\% \text { correct answers } \\
\text { on the right }+\mathrm{CS}\end{array}$ & SVQ & $\begin{array}{l}\text { VHIT } \\
\text { (Al) }\end{array}$ \\
\hline$M, 78$ & 80 & 50 & 75 & 41,9 & 32 & $\begin{array}{l}45 \% \\
\mathrm{R}\end{array}$ \\
\hline M, 42 & 66,7 & 20 & 57,9 & 15,3 & 39 & $\begin{array}{l}28 \% \\
\mathrm{R}\end{array}$ \\
\hline$F, 39$ & 100 & 44,4 & 95 & 36,9 & 44 & $\begin{array}{l}62 \% \\
\mathrm{R}\end{array}$ \\
\hline$M, 61$ & 60 & 10 & 50 & 9 & 51 & $\begin{array}{l}55 \% \\
\mathrm{R}\end{array}$ \\
\hline Mean & 78,12 & 39,81 & 72,2 & 33,0 & & \\
\hline
\end{tabular}


Table 3

Control group (CTRL): demographic data, distribution of percentage of correct answers (\%CA) on the left and on the right side, without and with confounding screen (CS), situational vertigo questionnaire (SVQ)

score

\begin{tabular}{|c|c|c|c|c|c|}
\hline $\begin{array}{l}\text { Sex, } \\
\text { Age }\end{array}$ & $\begin{array}{l}\text { \% correct } \\
\text { answer on the } \\
\text { left }\end{array}$ & $\begin{array}{l}\% \text { correct answer } \\
\text { on the right }\end{array}$ & $\begin{array}{l}\% \text { correct answer } \\
\text { on the left + CS }\end{array}$ & $\begin{array}{l}\% \text { correct answer on } \\
\text { the right + CS }\end{array}$ & SVQ \\
\hline$F, 45$ & 100 & 100 & 100 & 100 & 0 \\
\hline$M, 66$ & 100 & 100 & 95 & 100 & 2 \\
\hline $\mathrm{M}, 48$ & 100 & 100 & 95 & 95 & 3 \\
\hline $\mathrm{F}, 49$ & 85 & 100 & 90 & 90 & 2 \\
\hline$M, 70$ & 90 & 100 & 100 & 90 & 5 \\
\hline $\mathrm{M}, 44$ & 90 & 100 & 100 & 100 & 4 \\
\hline$M, 68$ & 100 & 100 & 90 & 100 & 7 \\
\hline $\mathrm{M}, 42$ & 100 & 100 & 95 & 100 & 2 \\
\hline$F, 50$ & 100 & 90 & 90 & 100 & 4 \\
\hline M, 58 & 100 & 100 & 81,8 & 88,9 & 8 \\
\hline$M, 63$ & 100 & 100 & 90 & 90 & 7 \\
\hline$F, 39$ & 100 & 100 & 100 & 100 & 3 \\
\hline$F, 66$ & 100 & 90 & 95 & 90 & 10 \\
\hline$F, 64$ & 100 & 100 & 95 & 100 & 4 \\
\hline$F, 54$ & 100 & 100 & 100 & 95 & 7 \\
\hline$F, 54$ & 100 & 100 & 92,9 & 100 & 8 \\
\hline$M, 65$ & 100 & 100 & 94,5 & 95 & 7 \\
\hline$F, 45$ & 90 & 70 & 90 & 100 & 12 \\
\hline$F, 39$ & 100 & 100 & 100 & 90 & 6 \\
\hline$M, 47$ & 100 & 100 & 100 & 100 & 4 \\
\hline Mean & 97,75 & 97,5 & 94,71 & 96,20 & \\
\hline
\end{tabular}

All the patients suffering from AUV, according with the initial diagnosis, showed a unilateral caloric weakness and a pathological Asymmetry Index on VHIT (11 case of left AUV and 9 cases of right AUV, in the text referred to as AUV-L and AUV-R respectively). Only two patients in the AUV group reported the presence of motion sickness. 
In VM group the \%CA at fHIT was $90,48 \%$ on the left side and $93,67 \%$ on the right side; with the confounding screen (optokinetic stimulation), the \%CA fall at $73,69 \%$ on the left side and $73,63 \%$ on the right side (Fig. 1). Considering \%CA on the left and on the right simultaneously, in VM groups the \%CA at fHIT was $92,07 \%$ without CS and $73,66 \%$ with CS. A statistically significant difference has been found between \%CA of VM group with and without CS ( $p<.001)$, between \% CA of VM group without CS and CTRL group without CS $(p<.01)$ and, mostly, between \%CA of VM group with CS and CTRL with CS ( $p$ $<.001)$. Meanwhile, no significant difference has been found between \%CA of CTRL group with and without CS (Fig. 1).

The \%CA in the AUV-R group was $78,12 \%$ on the left and $39,81 \%$ on the right without CS; with CS the \%CA was $72,23 \%$ on the left and $32,97 \%$ on the right. In the AUV-L group the \%CA without CS was $53,60 \%$ on the left and $85,30 \%$ on the right without CS; with CS the \%CA was $40,25 \%$ on the left and $71,28 \%$ on the right. In the control group the \%CA was $97,75 \%$ on the left and $97,5 \%$ on the right without CS and $94,71 \%$ on the left and $96,19 \%$ on the right with CS. No significant difference has been found between left and right \%CA without and with CS, in between the VM and CTRL groups (Fig. 2). Furthermore, a significant difference has been found between \%CA on the deficit side and the normal side in AUV-L and AUV-R groups, both without CS $(p<.001$ and $p<.001$, respectively) and with $C S(p<.001$ and $p<.001$, respectively). A significant difference has been found also in \%CA in AUV-R and AUV-L on the normal and on the deficit side and \%CA in CTRL group without CS $(p<.05, p<.05$ and $p<.001, p<.001$, respectively), and with CS ( $p<.01, p<.01$ and $p<.001, p<.001$, respectively) (Fig. 2). The data relating to the tests between groups confirm the result of the analysis of variance which allows us to reject the null hypothesis that the subjects of the study are from the same population, but also allow us to say that the population of migraineurs and that of vestibular deficits are among them distinct $(p<.001)$. Since the data are normally distributed, it is possible to establish limits of normality of the response (average plus twice the standard deviation): it therefore appears that if the absolute value of the difference of the errors with and without confounding screen ( $\Delta C S$ ) exceeds 10,09 , the answer is to be considered pathological.In the control group, only in three cases (out of 40) the absolute value of $\Delta \mathrm{CS}$ is higher than $10,09(7,5 \%)$. On the other hand, among patients with VM, 29 out of 50 are pathological (58\%).Among patients with unilateral vestibular deficit, 10 out of 40 tests are pathological (25\%).

The mean $\Delta$ CS value in VM group is 18,36 . Then, $\Delta C S$ in CTRL group is 2,15. Finally, $\Delta$ CS in AUV group is 10,4 (Fig. 3). A statistically significant difference has been found between $\triangle \mathrm{CS}$ in VM and CTRL groups $(p<0.001)$, and $\triangle C S$ in AUV and CTRL groups $(p<0.001)$. Further, a lower significant difference has been found between $\triangle C S$ in VM and AUV groups $(p<0.05)$ (Fig. 3).

Regarding the report of SVQ, the mean score of VM group is 40.36 (from 21.0 to 61.0 ); in the AUV the mean score is 38.35 (from 28.0 to 53.0); in the control group, instead, the mean score is 5.25 (from 0.0 to 12.0). The difference between groups is statistical significative $(p<.001)$. A significant difference has been found between SVQ of VM and the AUV patients with the control group $(p<.001$ and $p<.001$, respectively). On the other hand, no significant differences have been found between VM and AUV at SVQ scores $(p=0.530)$. (Fig. 4). 


\section{Discussion}

To achieve an adequate spatial orientation, the integration of vestibular, proprioceptive and visual inputs is mandatory. Some normal subjects show an overreliance on visual stimuli probably due to a lack of confidence in vestibular or somatosensory input [8]. This condition is termed VD and could be considered a perceptual trait or cognitive style variably expressed in the general population $[20,21]$ and more recently was named by Barany Society as visual induced dizziness [22]. In patients suffering from vestibular disease, the interactions between visual and vestibular input could be impaired $[4,11,23]$ and for this reason a complex visual input could induce a postural destabilization [24]. In this situation the inability to ignore a moving and/or complex visual scene (optokinetic stimuli) can be confused as a self-motion resulting in instability. Following AUV, VD could be a strong predictor of poor clinical outcome [9]; patients with VM show evidence suggesting an increased visual dependence, as measured with an optokinetic rotating disc [11,25-27]. Recently a new method to evaluate the functional performance of the vestibuloocular reflex (VOR) during passive head movement has been proposed: the fHIT quantifies the percentage of correctly recognized optotypes using a relatively large, fixed-size optotype) during head impulses scanning a wide range of head angular accelerations [18]. The fHIT identifies how much the actual stabilization ability is impaired as head acceleration increase [15] and combining this test with an optokinetic stimulation (rotating background, confounding screen), an impaired integration between visual and vestibular stimuli can be detected [13].

Our results indicated that a significant percentage (58\%) of patients with VM showed a significant decrease of the \%CA using a confounding screen (o-fHIT) as compared with normal subjects. Also, in AUV patients this behavior can be detected; but in the latter, there is a clear difference according with the affected side. The reduction of \%CA passing from a normal to a confounding screen determinate a reduction of the results only on the affected side. In other term the deterioration of the performance on fHIT passing from a normal to a confounding screen is not side-dependent in VM while in AUV patients the reduction of the \%CA using an optokinetic stimulation is detected mostly when rotating the head towards the affected side. In these patients, the abnormalities of the results of o-fHIT on the affected side is clearly related to an altered gaze control as a consequence of a reduction of the VOR gain. On the contrary, the impairment of the multisensory integration showed by VM patients during fHIT with optic flow stimulation, could be considered as consequence of a visuo-vestibular conflict related to central nervous system abnormalities, frequently encountered in these kind of disease [28,29]. Versino et al [13] reported a functional vestibular impairment using the fHIT especially when head impulse test is performed in combination with optokinetic stimulation. Our results seem to confirm this observation: our group of AUV patients showed, as expected, a reduced capability to correctly recognize a visual target during $\mathrm{fHIT}$ when the head is turned to the affected side. This impairment slightly increases when an integration of visuo-vestibular input is necessary as happens when an optokinetic stimulus is presented so inducing a decrease of the \%CA. In VM patients our results indicate that the \%CA is normal in most of the subjects and only using the optokinetic stimulation this parameter strongly decrease (with no difference between the two sides) in respect not only to the control group but also in comparison with the AUV group showing that visual dependence is the cause of the decay of the responses when the 
background is in motion. Recently, Al-Sharif et al [30] studied the effects of visual-vestibular mismatch using a specific questionnaire and a computerized Rod and Frame test; they reported that the presence of VD is significantly higher in patients suffering from headache and dizziness than in subjects with headache only. Our results are in line with this observation indicating a very important role of the vestibular involvement in VM causing a difficulty in resolving conflicting visuo-vestibular signals, as demonstrated by the significant difference in delta CS between our three group of subjects. Another result of our study is represented by the high percentage (\%) of patients with VM suffering from motion sickness in respect to the AUV patients or controls. Probably motion sickness and the reduced capability of integrate visual vestibular signals (as happens in o-f-HIT) have a common denominator: a sensory conflict could modify the awaited experiences causing a discordance between the expected and the real perception leading to motion sickness or visual vertigo or dizziness [31,32].

In our study the evaluation of the patients' self-feeling was conducted using the Italian version of SVQ [19]. This questionnaire was introduced by Jacob et al. in 1993 for quantifying space and motion discomfort in patients suffering from anxiety and/or balance disorders and subsequently adapted to evaluate the presence of visual vertigo [4]. Our results regarding SVQ indicate that visual induced vertigo and dizziness are very common both in VM and AUV patients. This results it is not surprising because migrainous patients (with and without associated vertigo) are highly susceptible to an increased visuovestibular conflict $[19,33,34]$. High SVQ levels were described in patients with vestibular disease $[4,8,11,35]$, and our results seems to confirm this observation. Our group of AUV patients were evaluated few days after the onset of the disease: probably this condition is the cause of the higher SVQ score encountered in our cohort. Surely the presence of an increased SVQ levels and the low \%CA with optokinetic stimulation could indicate the possibility of an evolution towards chronic symptoms after AUV and could identify the need of an early rehabilitation treatment using desensitization technique $[9,36]$, but more pregnant data should be obtained in a more advanced stage of the disease. In other term, our results indicate that symptoms of visual vertigo or impaired visuo-vestibular interactions are very frequent in the subacute phase of AUV with a similar rate as encountered in some patients suffering from VM. In a high percentage of patients suffering from AUV, the process of vestibular compensation could induce a reduction of this kind of symptoms, but its persistence is significantly higher in those with worse clinical outcome [9].

Another important topic is the correlation between the presence of psychopathological conditions (anxiety/depression) and impaired visuo-vestibular interactions. Previous studies reported conflicting results[4,37], but recently Teggi et al [38] studied 25 patients diagnosed as affected from PPPD showing that fHIT with optokinetic stimulation provoked more readings error than in controls, supporting the hypothesis that increased anxiety could lead to an alteration of visuo-vestibular interactions. This observation could introduce a bias in our study considering that we do not evaluate with established questionnaire the levels of anxiety/depression in our cohort of patients. For this reason, we consider this aspect as one of the study limitations in our manuscript. However, the presence of some psychopathological aspects in VM is very common so much that a combination of a balance disorder, migraine, and anxiety has named migraine anxiety-related dizziness [39] based on shared mechanisms in 
afferent interoceptive information and central nervous system processing [40]. Another study limitation is represented by characteristics of the study design: a prospective study with a large cohort of patients will surely bring a better assessment the role of fHIT in diagnosing visual dependence in subjects suffering from VM and AUV and in evaluating the efficacy of rehabilitative and/or pharmacological treatment.

\section{Methods}

Between December 2018 and June 2020, we consecutively recruited 25 patients fulfilling the diagnostic criteria for VM [5]. All the VM patients were evaluated in the intercritical phase and in absence of any actual pharmacological prophylactic treatment. All patients also underwent a complete vestibular evaluation, which involves bedside examination, caloric reflex test, the video head impulse test (VHIT) and functional head impulse test (fHIT). In the same period, we recruited 20 patients suffering from AUV. We diagnosed AUV as a syndrome characterized by rapid onset of severe dizziness without neurologic or audiologic symptoms, unidirectional horizontal nystagmus, unilateral vestibular areflexia/hyporeflexia on bithermal caloric test, and positive head impulse test result in the direction opposite to the fast phase of the nystagmus. Each patient with AUV underwent to the vestibular instrumental examination (bithermal caloric test and video Head Impulse Test, vHIT) after the disappearance of the acute symptomatology within 8-14 days after the onset of the symptoms. The vHIT was performed by employing a dedicated device (ICS Impulse System; GN Otometrics): the patient was asked to stare at an Earth-fixed target (3-cmdiameter spot located $1.5 \mathrm{~m}$ in front); then, 20 low-amplitude $\left(10^{\circ}-20^{\circ}\right)$ but high-velocity head impulses (150-200 deg/s) were randomly administered on each side for every semicircular canal. The device software automatically calculates the average high-velocity VOR gain. The software also calculates the asymmetry index (Al\%, normal values within 15\% [\% confidence limit $0-15 \%]$; this value resulted from our own data collected on a group of 50 normal subjects age ranging 20 to 80 ) between the right and left sides. Al was calculated as $\left|G A=\left[\frac{\left(G_{L}-G_{R}\right)}{\left(G_{L}+G_{R}\right)}\right] \times 100\right|$ where $G R$ denotes right sided mean gain and $G L$ denotes left sided mean gain. The Al\% shows differences between the sides in terms of high-velocity VOR [17]. All the patients underwent to cranial MRI investigation to rule out a central nervous system involvement. Patients suffering from Menière's Disease, sudden sensorineural hearing loss or recent head trauma were excluded. Altogether we considered a control group consisting of 20 healthy subjects, excluding subjects who suffered in the past from AUV and who did report recent episodes of benign paroxysmal positional vertigo. They also must not report a personal or familiar history of migraine. All the subjects underwent to fHIT using the Beon Solution System ${ }^{\circledR}$ (Beon Solution srl, Treviso, Italy): the patient was seated 1.5 meters away from the computer screen (Full HD, resolution 1920x1080, refresh rate $60 \mathrm{~Hz}$ ) and was wearing an accelerometer mounted on the forehead through a tape. Landolt's Optotype $\mathrm{C}$ was used and first of all the examination acuity was evaluated, progressively decreasing the size of the symbols: in this way the dimensions of the visual stimulus were normalized according to the characteristics of the subject examined. Subsequently, the examiner quickly moves the patient's head on the horizontal plane (at least ten turns in each direction), with an acceleration ranging between 3000 and $6000 \% \mathrm{~s}^{2}$. During the movement (as soon as the acceleration reached a limit set by the manufacturer) a 
symbol was displayed on the monitor for a time of 33 milliseconds [18]. The patient was asked to recognize the symbol that appeared on the monitor during head movement and to press the equivalent key. The parameter taken into consideration was the average percentage of correct answers (\%CA) with respect to the total of answers presented for each frequency band examined (acceleration bins). The test was then repeated with the addition of confounding screen (CS) that is a moving background constituted by a rotating cloud of yellow dots, named optokinetic fHIT (o-fHIT) displayed on the screen while the above-described test is being performed. To evaluate the results of the test, the following procedure was carried out:1) the answers for the head rotation to the right and those for the rotation to the left were separated and considered different tests: in this way the number of exams becomes double compared to the number of patients; 2) For each test, the difference in \%CA without and with confounding screen was evaluated.

All the subjects were submitted to the Italian version of the Situational Vertigo Questionnaire (SVQ) [19] to identify if vertigo symptoms are provoked or exacerbated by specific disorienting visual context. The total score will be then calculated as the sum of single item scores divided per 19 minus the number of never experienced situations (total score/19-number of "never experienced" answer).

Ethical review and approval by the local Institutional Board (Comitato Etico Azienda OspedalieroUniversitaria Pisana, Pisa, Italy) were waived for this study. Due to its retrospective nature, it not being set up as part of a research project. Furthermore, the study does not include new experimental diagnostic protocols and the patients included in the study were diagnosed according to national guidelines. Written informed consent was obtained from all participants, and the study was conducted in accordance with the 1964 Declaration of Helsinki.

\section{Statistical study}

The data obtained were statistically compared with the variance analysis method. The values obtained are distributed in a normal way, therefore it was possible to perform:

1) a study of variance (one-way ANOVA), which allowed to reject the null hypothesis of homogeneity of the groups $(p<0.05)$.

2) A statistical comparison between patients with migraine vertigo (one-way t-test) and normal group;

3) A statistical comparison between patients with compensated acute vestibular deficit and the normal group (one-way t-test);

4) A comparison between patients suffering from migraine and those suffering from compensated acute vestibular deficit (one-way t-test).

A study of variance with Tukey's correction was also carried out, which was also significant due to the rejection of the null hypothesis between the groups $(p<0.05)$.

\section{Declarations}

Author Contributions: "Conceptualization, A.P.C., N.D. and N.V.; methodology, A.P.C. and F.L.; software, F.L. O.M.; validation, A.P.C., O.M and F.L.; formal analysis, F.L., A.P.C.; investigation, A.P.C., N.D. and N.V.; data 
curation, N.D. and N.V.; writing-original draft preparation, A.P.C.; writing-review and editing, A.P.C., O.M. N.D.; supervision, A.P.C. All authors have read and agreed to the published version of the manuscript."

Funding: This research received no external funding

Institutional Review Board Statement: Ethical review and approval by the local Institutional Board (Comitato Etico Azienda Ospedaliero-Universitaria Pisana, Pisa, Italy) were waived for this study. Due to its retrospective nature, it not being set up as part of a research project. Furthermore, the study does not include new experimental diagnostic protocols and the patients included in the study were diagnosed according to national guidelines.

Informed Consent Statement: Written informed consent has been obtained from the patient(s) to publish this paper" if applicable.

Data Availability Statement: The data presented in this study are available on request from the corresponding author. The data are not publicly available due to privacy restrictions.

Conflicts of Interest: The authors declare no conflict of interest.

\section{References}

1. Kerber, K.A. Acute vestibular syndrome. Semin Neurol. 40, 59-66 (2020).

2. Formeister, E.J., Rizk, H.G., Kohn, M.A. \& Sharon, J.D. The epidemiology of vestibular migraine: a population-based survey study. Otol Neurotol. 39, 1037-1044, (2008).

3. Neuhauser, H.K. et al. Migrainous vertigo prevalence and impact on quality of life. $67,1028-1033$ (2007).

4. Guerraz, M. et al. Visual vertigo: symptom assessment, spatial orientation and postural control. Brain, 124, 1646-1656 (2001).

5. Lempert, T. et al. Vestibular migraine: diagnostic criteria. J Vest Res. 22, 167-172 (2012).

6. R.W. Vestibular Migraine I: Mechanisms, Diagnosis, and Clinical Features. Semin Neurol. 40, 76-82 (2020).

7. Lempert, T. \& Von Brevern, M. Vestibular Migraine. Neurol Clin., 37, 695-706 (2019).

8. Bronstein, A.M. Visual vertigo syndrome: clinical and posturography findings. J Neurol Neurosurg Psychiatry. 59, 472-476 (1995).

9. Cousins, S. et al. Visual dependency and dizziness after vestibular neuritis. PLoS ONE. 9: e105426 (2014).

10. Maire, R. et al. Discussion about visual dependence in balance control. European Society for clinical evaluation of balance disorders. J Int Adv Otol. 13, 404-406 (2017).

11. Agarwal, K. et al. Visual dependence and BPPV. J Neurol. 259, 1117-1124 (2012). 
12. Obermann, M. et al. Central vestibular system modulation in vestibular migraine. $34,1053-1061$ (2014).

13. Versino, M. et al. The integration of multisensory motion stimuli is impaired in vestibular migraine patients. J Neurol. 267, 2842-2850 (2020).

14. Witkin, H. A. \& Asch S.E. Studies in space orientation. IV: Further experiments on perception of the upright with displaced visual fields. J Exp Psychol. 38, 762-782 (1948).

15. Versino, M. et al. Reading while moving: the functional assessment of VOR. J Vestib Res. 24, 459464 (2014).

16. Corallo, G., Versino, M., Mandala, M., Colnaghi, S. \& Ramat, S. The functional head impulse test: preliminary data. J Neurol. 265, 35-39 (2018).

17. Halmagyi, G.M. et al. The video head impulse test. Front Neurol. 258. doi:3389/fneur.2017.00258 (2017).

18. Colagiorgio, P., Colnaghi, S., Versino, M. \& Ramat, S. A New Tool for Investigating the Functional Testing of the VOR. Front Neurol. 4, 165 (2013).

19. Colnaghi, S. et al. Validation of the Italian version of the Dizziness Handicap Inventory, the situational vertigo questionnaire, and the activity-specific balance confidence scale for peripheral and central vestibular symptoms. Front Neurol. 8, 528 (2017).

20. Witkin, H.A. \& Goodenough, D.R. Cognitive styles: Essence and origins. (International University Press: New York, USA, 1981).

21. Roberts, R.E., Da Silva Melo, M., Siddiqui, A.A., Arshad, Q. \& Patel M. Vestibular and oculomotor influences on visual dependency. J Neurophysiol. 116, 1480-1487 (2016).

22. Bisdorff, A., von Brevern, M., Lempert, T. \& Newman-Toker, D.E. (on behalf of the Committee for the Classification of Vestibular Disorders of the Barany Society), Classification of vestibular symptoms: Towards an international classification of vestibular disorders. J Vest Res. 19, 1-13 (2009).

23. Bronstein, A.M. Visual and psychological aspects of vestibular disease. Curr Opin Neurol. 15, 1-3 (2002).

24. Bronstein, A.M. Multisensory integration in balance control. Handb Clin Neurol. 137, 57-66 (2016).

25. Furman, J.M., Sparto, P.J., Soso, M. \& Marcus, D. Vestibular function in migraine-related dizziness: a pilot study. J Vest Res. 15, 327-332 (2015).

26. Lempert, T. Vestibular migraine. Semin Neurol. 33, 212-218 (2013).

27. Bednarczuk, N.F. et al. Abnormal visuo-vestibular interactions in vestibular migraine: A cross sectional study. 142, 606-616 (2019).

28. Shin, J.H., Kim, Y.K., Kim, H.J. \& Kim, J.S. Altered brain metabolism in vestibular migraine: comparison of interictal and ictal findings. 34, 58-67 (2014).

29. Espinosa-Sanchez, J.M. \& Lopez-Escamez, J.A. New insights into pathophysiology of vestibular migraine. Front Neurol. 6, 12 (2015). 
30. Al Sharif, D.S., Roehm, P., Lidemann, T.L., Dumenci, L. \& Keshner, E.A. Visual-vestibular mismatch correlates with headache. J Vest Res. 10.3233/VES-201539 (2021).

31. Murdin, L. et al. Motion sickness in migraine and vestibular disorders. J Neurol Neurosurg Psychiatry. 86, 585-587 (2015).

32. Abouzari, M. et al. The Relationship BetweenVestibular Migraine and Motion Sickness Susceptibility. Otol Neurotol. 41,1116-1121 (2020).

33. Teggi, R et al. Clinical features, familial history, and migraine precursors in patients with definite vestibular migraine: The VM- Phenotypes Projects. 58, 534-544 (2018).

34. Beh, S.C., Masrour, S., Smith, S.V. \& Friedmann, D.I. The spectrum of vestibular migraine: Clinical features, triggers, and examination findings. 59, 727-740 (2019).

35. Jacob, R.G. et al. Discomfort with space and motion: a possible marker of vestibular dysfunction assessed by the Situational Characteristics Questionnaire. J Psychopathol Behav Assess. 15, 299324 (1993).

36. Pavlou, M. The Use of Optokinetic Stimulation in Vestibular Rehabilitation. J Neurol Phys Ther. 34, 105-110 (2010).

37. Zur, O. et a. Anxiety among individuals with visual vertigo and vestibulopathy. Disabil Rehabil. 37, 2197- 2202 (2015).

38. Teggi, R., Gatti, O., Cangiano, J., Fornasari, F. \& Bussi, M. Functional head impulse test with and without optokinetic stimulation in subjects with persistent postural perceptual dizziness (PPPD): preliminary report. Otol Neurotol. 41, e70-e75 (2020).

39. Furman, J.M., Balaban, C.D., Jacob, R.G. \& Marcus, D.A. Migraine-anxiety related dizziness (MARD) : A new disorder? J Neurol Neurosurg Psychiat. 76, 1-8 (2005).

40. Balaban, C.D., Jacob, R.G. \& Furman, J.M. Neurologic bases for comorbidity of balance disorders, anxiety disorders and migraine: neurotherapeutic implications. Expert Rev Neurother. 11, 379-394 (2011).

\section{Figures}




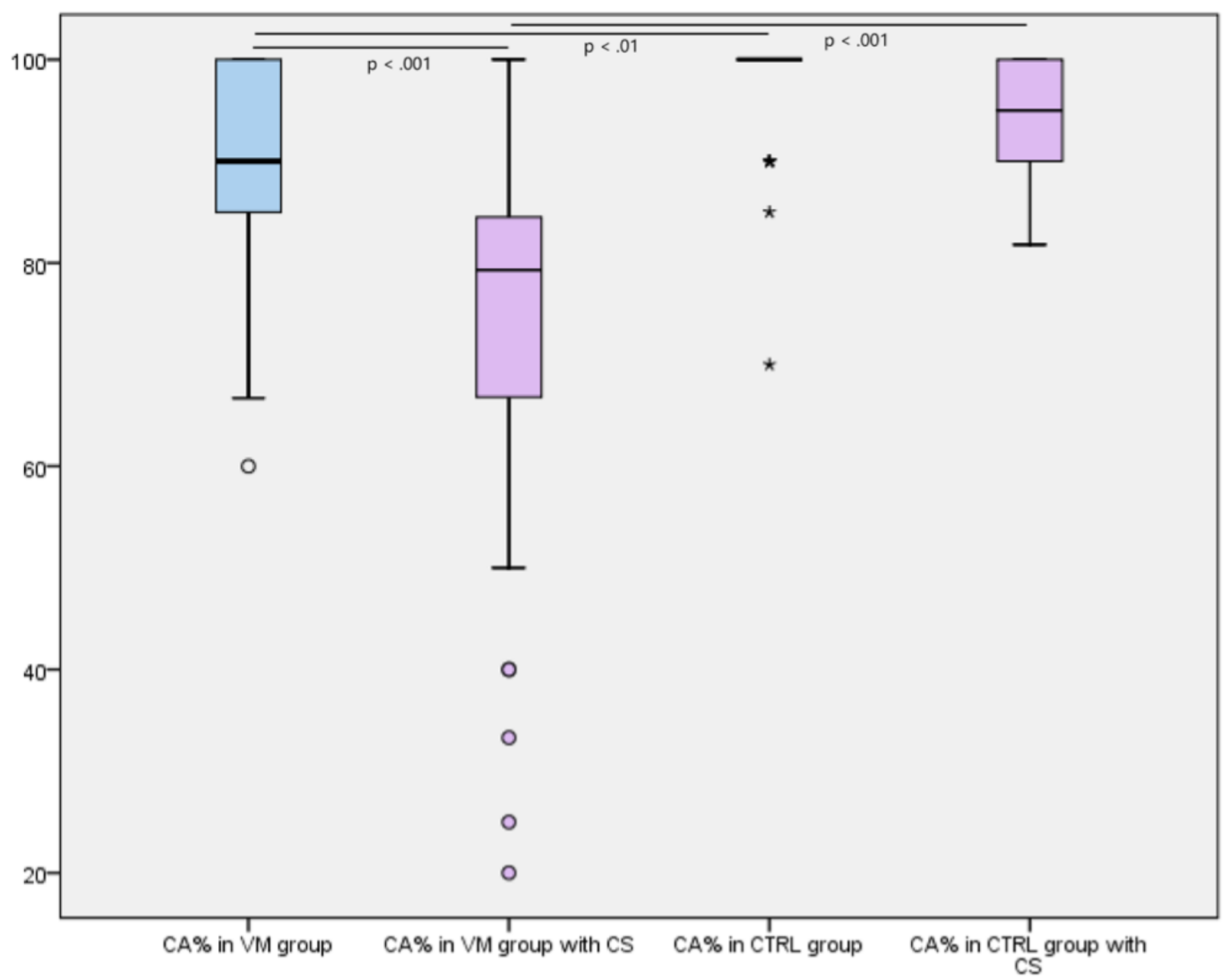

\section{Figure 1}

Boxplot showing the percentage of correct answer at fHIT in VM group and CTRL group, without and with the confounding screen. A statistically significant difference has been found between \%CA of VM group with and without CS $(p<.001)$, between \% CA of VM group without CS and CTRL group without CS $(p<$ $.01)$ and, mostly, between \%CA of VM group with CS and CTRL with CS $(p<.001)$. No significant difference has been found between \%CA of CTRL group with and without CS. 


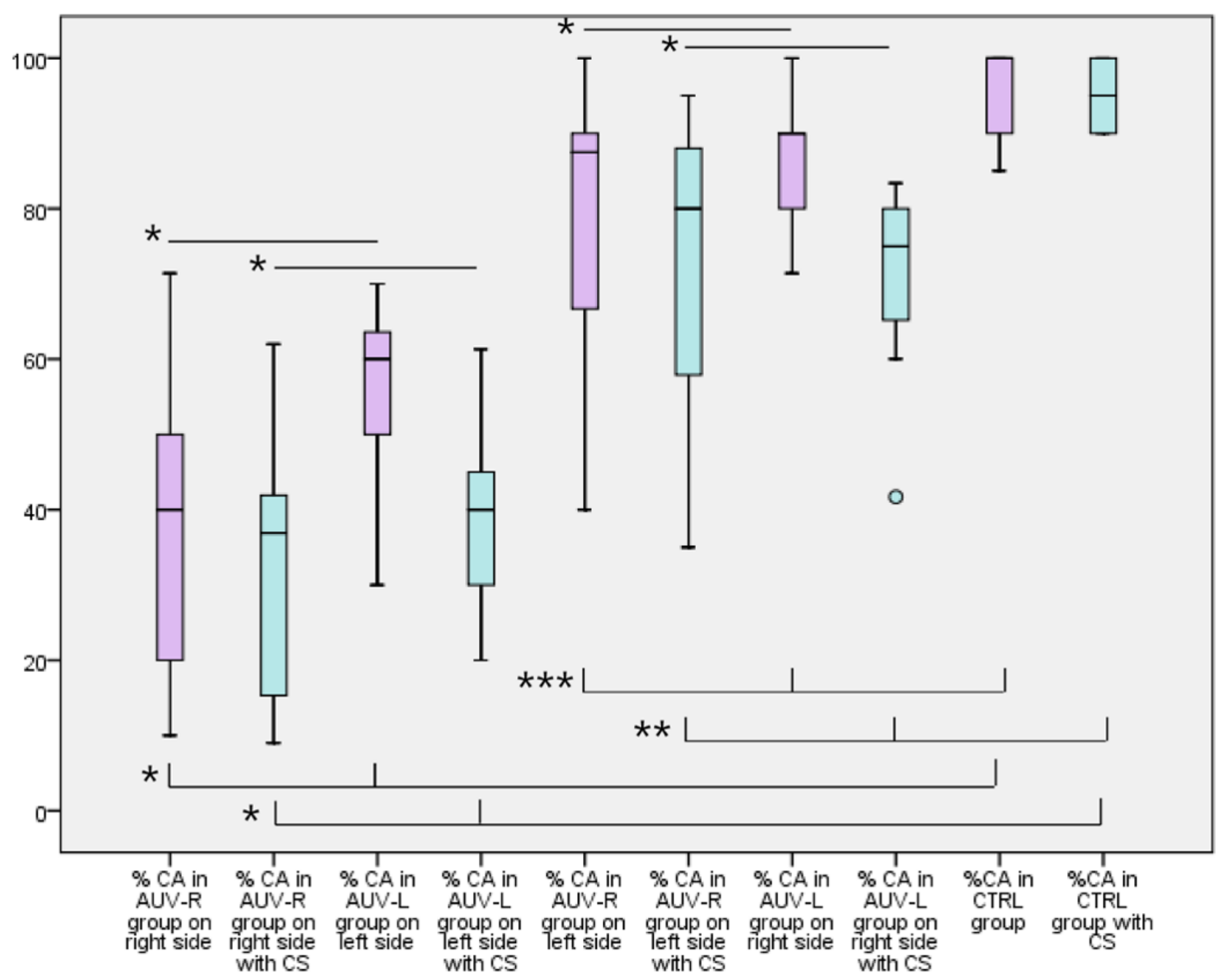

Figure 2

Boxplot showing the percentage of correct answer in the AUV group on the right and left side, without and with the confounding screen and the \%CA in the control group without and with confounding screen. $\mathrm{A}$ significant difference has been found between \%CA on the deficit side and the normal side in AUV-L and AUV-R groups, both without CS ( $p<.001$ and $p<.001$ [*], respectively) and with CS $(p<.001$ and $p<.001$ $[*]$, respectively). A significant statistically difference has been found also in \%CA in AUV-R and AUV-L on the normal and on the deficit side and \%CA in CTRL group without CS $(p<.05, p<.05[\star \star \star]$ and $p<.001, p$ $<.001$ [*], respectively), and with CS $(p<.01, p<.01[* *]$ and $p<.001, p<.001[*]$, respectively). 


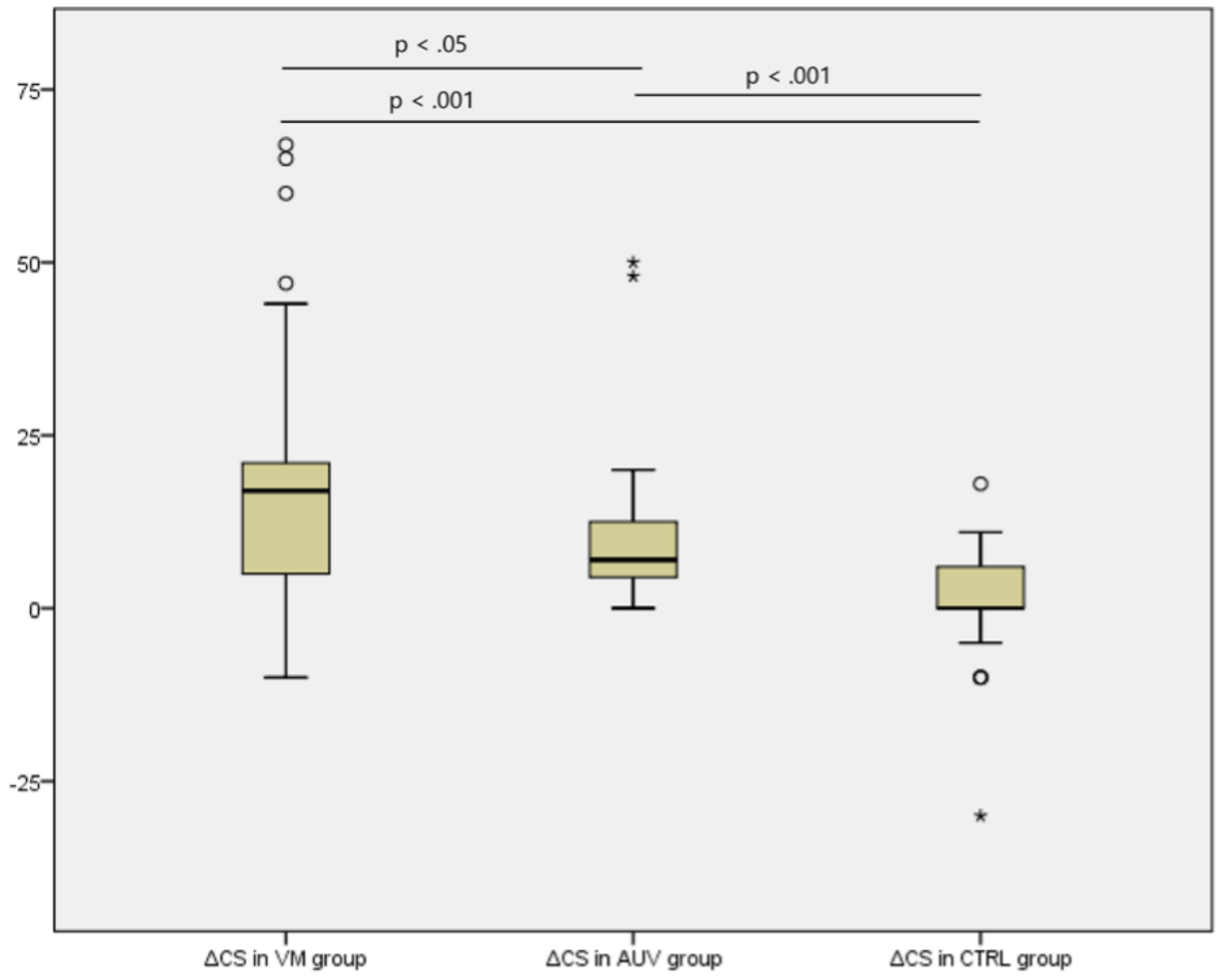

Figure 3

Boxplot showing the $\triangle \mathrm{CS}$ (the absolute value of the difference of the errors with and without confounding screen) in VM, AUV and CTRL group. A statistically significant difference has been found between $\triangle C S$ in VM and CTRL groups $(p<0.001)$, and $\triangle C S$ in AUV and CTRL groups $(p<0.001)$ and between $\triangle C S$ in VM and AUV groups $(p<0.05)$. 


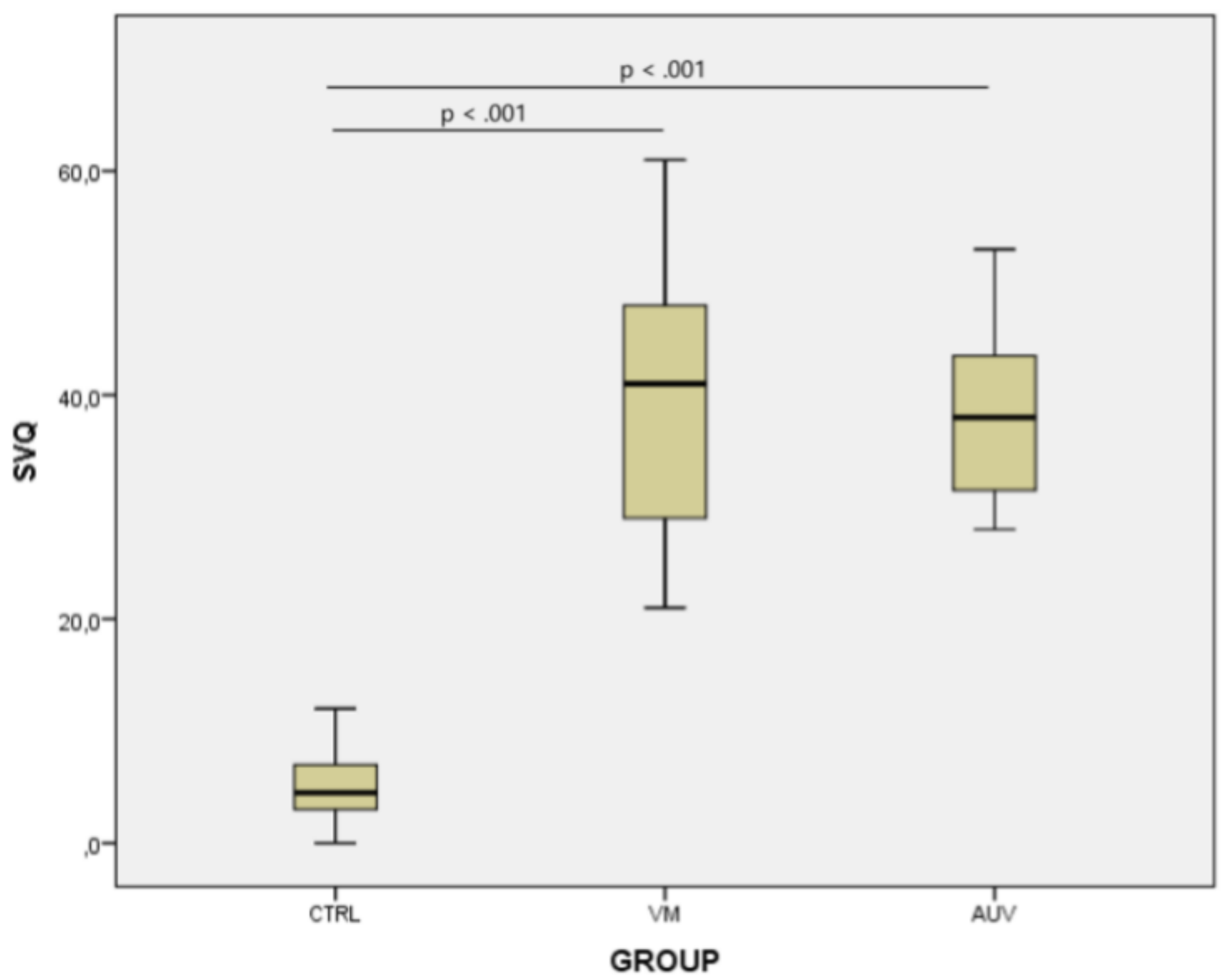

Figure 4

Boxplot showing the SVQ score in the VM, AUV and CTRL group. A significant difference has been found between SVQ of VM and the AUV patients with the control group ( $p<.001$ and $p<.001$, respectively). No significant differences have been found between VM and AUV at SVQ scores. 